\title{
Implicit Optimality Criterion for Convex SIP problem with Box Constrained Index Set
}

\author{
Kostyukova O.I.* Tchemisova T.V. ${ }^{\dagger}$
}

\begin{abstract}
We consider a convex problem of Semi-Infinite Programming (SIP) with a multidimensional index set defined by a finite number of box constraints. In study of this problem we apply the approach suggested in [20] for convex SIP problems with one-dimensional index sets and based on the notions of immobile indices and their immobility orders. For the problem under consideration we formulate optimality conditions that are explicit and have the form of criterion. We compare this criterion with other known optimality conditions for SIP and show its efficiency in the convex case.
\end{abstract}

Key words. Semi-Infinite Programming (SIP), Semidefinite Programming (SDP), constraint qualifications (CQ), immobile index, optimality conditions.

AMS subject classification. 90C25, 90C30, 90C34

\section{Introduction}

Consider an optimization problem in the form

$$
\begin{array}{ll} 
& (P) \quad \min _{x \in \mathbb{R}^{n}} c(x) \\
\text { s.t. } & x \in X:=\left\{x \in Q \subset \mathbb{R}^{n}: f(x, t) \leq 0, \forall t \in T\right\},
\end{array}
$$

where $T \subset \mathbb{R}^{s}$ is a given index set, $s \geq 1 ; Q$ is some subset of $\mathbb{R}^{n}$, and functions $c(x), f(x, t)$, are defined for $x \in Q, t \in T$.

When the index set $T$ in problem $(P)$ is infinite, this problem is referred to as that of SemiInfinite Programming (SIP). Notice that the set $T$ can be one dimensional $(T \subset \mathbb{R})$ or multidimensional $\left(T \subset \mathbb{R}^{s}, s>1\right)$. If the objective function $c(x)$ is a convex function and the feasible set $X$ is convex in $\mathbb{R}^{n}$, then problem $(P)$ is convex.

Having appeared as a new field of Optimization in 70-es in the works of A. Charnes, R. Hettich, K.O. Kortanek, H.Th. Jongen, and others (see [6, 11, 13, 29] et al), Semi-Infinite Programming has always attracted special interest due to its practical applications and relationship with other

\footnotetext{
*Institute of Mathematics, National Academy of Sciences of Belarus, Surganov str. 11, 220072, Minsk, Belarus (kostyukova@im.bas-net.by).

${ }^{\dagger}$ Mathematical Department, University of Aveiro, Campus Universitario Santiago, 3810-193, Aveiro, Portugal (tatiana@ua.pt).
} 
mathematical fields (for the references see [12, 18, 23, 31]). Many papers have been dedicated to the theory of SIP $([9,12,28])$, in general, and to optimality conditions, in particular. We refer to $[2,13,16,17,25,27]$ for the first-order and to $[3,14,19,26]$ for the second-order optimality conditions. The papers $[2,8,15,22]$ are especially devoted to convex SIP problems, and [4, 5] to abstract convex problems (finite and infinite).

Usually, when optimality conditions for some optimization problem are considered, a special attention is given to those that do not require additional assumptions about the constraints, so called constraint qualifications (CQ). This is due to the fact that the known CQs do not always hold for finite-dimensional and especially infinite-dimensional convex programs (see examples in $[2,15,22]$ et al.). Thus it was noticed by many authors that given an optimization problem in the form $(P)$, various CQs may fail in the presence of the constraints that vanish for any feasible solution (see [1, 7, 20,30], et al.). In different papers such constraints are differently referred to (see [21]). To avoid confusion, further in the paper we will use the following notation.

Definition 1. An index $t \in T$ is called immobile w.r.t. constraints of problem $(P)$ if $f(x, t)=0$ for all feasible solutions $x \in X$.

In our study of convex SIP problems, we are especially interested in the role that the immobile indices play in formulation of optimality conditions without CQ. In [19, 20] we considered convex SIP problems with one-dimensional index set and showed that immobile indices and their immobility orders represent objective and substantial characterizations of the feasible sets. We have proved new constructive optimality conditions that do not require any CQ and compared them with some other known CQ-free optimality conditions. The results of the comparison indicated the effectiveness of the new approach (see [19]). In [21], this approach was efficiently applied to study of linear semidefinite problems.

This paper is the first attempt to generalize the results obtained for SIP problems with onedimensional index set $T \subset \mathbb{R}$ to the case of problems with multidimensional index set $T \subset \mathbb{R}^{s}$, $s \geq 2$. The main objectives here are to expand on these problems the definitions of immobile indices and the corresponding immobility orders, and to formulate new efficient optimality conditions using the approach suggested in [19, 20].

We are concentrated on the study of problems whose constraints are convex w.r.t. the decision variable. For these problems, we show that the emptiness of the set of immobile indices is equivalent to the Slater type CQs. Given an immobile index, we introduce the corresponding set of feasible directions and define the directional immobility orders. For a particular case of SIP problem with a constraint function that is linear w.r.t. decision variable $x$, we prove an Implicit Optimality Criterion that provides optimality conditions in terms of such conditions for a special auxiliary problem. The constraints of this auxiliary problem are determined by multidimensional immobile indices and their orders. We show that the Implicit Optimality Criterion permits to deduce new explicit optimality conditions for convex SIP. All optimality conditions formulated in the paper are proved under an assumption that the orders of all immobile indices are not greater than one. We show that this assumption is less restrictive than some known Slater type CQ and that when it is relaxed, the proposed optimality conditions continue to be valid in their sufficient part. The comparison of the results of the paper with some known optimality results indicates that the immobility of indices is an important phenomenon in SIP which allows to better understand the structure of feasible sets and provides new techniques for formulation and proof of new CQ-free optimality conditions. 
The paper is organized as follows. In Section 2, we define immobile indices for SIP problems with multidimensional index sets and formulate different CQs that are equivalent. In Section 3, we consider a convex SIP problem with linear w.r.t. variable $x$ constraints and a box constrained index set $T \subset \mathbb{R}^{s}, 1<s<\infty$. For this problem, we concretize the basic notions and definitions and prove the Implicit Optimality Criterion. In Section 4, we derive new explicit optimality conditions (necessary and sufficient), suggest a simple illustrative example of their application, and show that in their sufficient part, these conditions continue to be valid even if we relax the assumption made at the beginning. Comparison of the optimality results obtained with other known optimality conditions is provided in Section 5. Some technical results are given in the Appendix.

\section{Immobile Indices and Constraint Qualifications in SIP}

Consider a convex SIP problem in the form $(P)$, where $Q \subset \mathbb{R}^{n}$ is a convex set, $T \subset \mathbb{R}^{s}$ is a compact index set; the functions $c(x)$ and $f(x, t)$, defined for $x \in Q, t \in T$, are convex w.r.t. $x$. As previously, let $X$ denote the feasible set of this problem:

$$
X=\{x \in Q: f(x, t) \leq 0, \forall t \in T\} .
$$

Consider the following CQs:

- The constraints (2) satisfy the Slater type condition I if

there exists $\bar{x} \in Q$ such that $f(\bar{x}, t)<0, \forall t \in T$.

- The constraints (2) satisfy the Slater type condition II if

$$
\begin{aligned}
& \text { for any index set }\left\{t_{i} \in T, i=1, \ldots, n+1\right\} \text { there exists } \\
& \text { a vector } \tilde{x} \in Q \text { such that } f\left(\tilde{x}, t_{i}\right)<0, i=1, \ldots, n+1 \text {. }
\end{aligned}
$$

Let

$$
\left\{\tau_{1}, \ldots, \tau_{n}\right\} \subset T
$$

be a set of indices from $T$. Consider the nonlinear problem

$\left(P_{D}\right)$

$$
\begin{gathered}
\min _{x \in Q} c(x) \\
\text { s.t. } f\left(x, \tau_{i}\right) \leq 0 \quad i=1, \ldots, n .
\end{gathered}
$$

In what follows, considering a problem $(A)$, we will denote by $\operatorname{val}(A)$ the optimal value of its objective function.

Under the Slater type condition II, the following proposition can be formulated.

Proposition 1. Consider the convex SIP problem $(P)$. Suppose that $X \neq \emptyset$ and let constraints (2) satisfy the Slater type condition II (3). Then there exists a set of indices (4) such that

$$
\operatorname{val}(P)=\operatorname{val}\left(P_{D}\right) .
$$


To prove Proposition 1 it is sufficient to repeat the proof of Proposition 5.105 from [3] replacing the condition $x \in \mathbb{R}^{n}$ by the following one: $x \in Q$ with convex $Q \subset \mathbb{R}^{n}$.

In the Introduction, we have defined the immobile indices for problem $(P)$. Let us denote this set by $T^{*}$ :

$$
T^{*}:=\{t \in T: f(x, t)=0 \forall x \in X\} .
$$

The following proposition is proved in [21].

Proposition 2. Let $X \neq \emptyset$ in the convex SIP problem $(P)$. Then the following conditions are equivalent:

CQ1: The constraints (2) satisfy the Slater type condition I.

CQ2: The constraints (2) satisfy the Slater type condition II.

CQ3: The set of immobile indices in problem $(P)$ is empty: $T^{*}=\emptyset$.

Let us suppose now that the index set $T$ of problem $(P)$ is a restricted box in $\mathbb{R}^{s}$ :

$$
T=\left\{t \in R^{s}: t=\left(t^{i}, i \in J\right), t_{*}^{i} \leq t^{i} \leq t^{* i}, i \in J\right\}, J=\{1, \ldots, s\},
$$

where $t_{*}^{i}<t^{* i}, i \in J$, are given real numbers.

For any $t \in T$, consider the sets

$$
J^{-}(t)=\left\{i \in J: t^{i}=t_{*}^{i}\right\}, J^{+}(t)=\left\{i \in J: t^{i}=t^{* i}\right\}, J(t)=\left\{i \in J: t_{*}^{i}<t^{i}<t^{* i}\right\} .
$$

A direction $l=\left(l^{i}, i \in J\right) \in \mathbb{R}^{s}$ is called feasible for an index $t \in T$ if

$$
l \in L(t):=\left\{l \in \mathbb{R}^{s}:\|l\|=1, l^{i} \geq 0 \text { if } i \in J^{-}(t), l^{i} \leq 0 \text { if } i \in J^{+}(t)\right\} .
$$

Suppose that the constraint function $f(x, t)$ of problem $(P)$ is sufficiently smooth w.r.t. $t$, i.e. all partial derivatives which occur in the sequel exist and are continuous.

Definition 2. Let us say that an immobile index $t \in T^{*}$ has the immobility order $q(t, l)$, $q(t, l) \in\{0,1, \ldots\}$, along a feasible direction $l \in L(t)$, if

1. $\left.\frac{d^{i} f(x, t+\alpha l)}{d \alpha^{i}}\right|_{\alpha=+0}=0, \forall x \in X, i=0, \ldots, q(t, l)$,

2. there exists a feasible point $\tilde{x}=x(t, l) \in X$ such that $\left.\frac{d^{(q(t, l)+1)} f(\tilde{x}, t+\alpha l)}{d \alpha^{(q(t) l)+1)}}\right|_{\alpha=+0} \neq 0$.

Here and in what follows we suppose that $\left.\frac{d^{0} f(x, t+\alpha l)}{d \alpha^{0}}\right|_{\alpha=+0}=f(x, t)$.

Consider an immobile index $t \in T^{*}$. Then by definition, vector $t$ solves the problem $\max _{\tau \in T} f(x, \tau)$ for all $x \in X$. Hence according to the first order necessary optimality condition for this problem we have

$$
\frac{\partial f^{T}(x, t)}{\partial t} e_{j}=\left.\frac{d f\left(x, t+\alpha e_{j}\right)}{d \alpha}\right|_{\alpha=+0}\left\{\begin{array}{l}
\geq 0, j \in J^{+}(t), \\
\leq 0, j \in J^{-}(t), \\
=0, j \in J(t),
\end{array} \quad \forall x \in X\right.
$$

where $e_{j}=\left(e_{j i}, i \in J\right)^{T}$ is the vector with components $e_{j i}=0$ if $i \neq j$ and $e_{j j}=1, j \in J$. Taking into account (7) and Definition 2 we conclude that

$$
q\left(t, \pm e_{j}\right)>0 \text { for } j \in J(t), t \in T^{*} .
$$


Denote

$$
\begin{gathered}
\bar{J}^{-}(t)=\left\{j \in J^{-}(t): q\left(t, e_{j}\right)=0\right\}, \bar{J}^{+}(t)=\left\{j \in J^{+}(t): q\left(t,-e_{j}\right)=0\right\}, \\
\bar{J}(t)=J \backslash\left(\bar{J}^{-}(t) \cup \bar{J}^{+}(t)\right), \quad t \in T^{*}
\end{gathered}
$$

It is easy to check that for $t \in T^{*}$, it holds

$$
\{l \in L(t): q(t, l)=0\}=L(t) \backslash \mathcal{M}(t),\{l \in L(t): q(t, l)>0\}=L(t) \cap \mathcal{M}(t),
$$

where $\mathcal{M}(t)$ is the subspace of $\mathbb{R}^{s}$ generated by the vectors $e_{j}, j \in \bar{J}(t)$. Consequently, taking into account (7) and (9), we conclude that for all $t \in T^{*}$, and for all $x \in X$, the following relations take place:

$$
\frac{\partial f^{T}(x, t)}{\partial t} e_{j} \leq 0, j \in \bar{J}^{-}(t) ; \quad \frac{\partial f^{T}(x, t)}{\partial t} e_{j} \geq 0, j \in \bar{J}^{+}(t) ; \frac{\partial f^{T}(x, t)}{\partial t} e_{j}=0, j \in \bar{J}(t),
$$

Having assumed that the set of immobile indices $T^{*}$ is finite, one can develop an algorithm that determines the immobile index set $T^{*}$ and the sets $\bar{J}^{ \pm}(t), \bar{J}(t)$, for all $t \in T^{*}$ on the base of the ideas from [20].

\section{Convex SIP problem with linear w.r.t. $x$ constraints}

\subsection{Definitions and basic notions}

Suppose that in (2), the set $Q$ coincides with $\mathbb{R}^{n}$ and the function $f$ is given by

$$
f(x, t)=\sum_{i=1}^{n} a_{i}(t) x_{i}+a_{0}(t)
$$

where $x=\left(x_{1}, \ldots, x_{n}\right)^{T} \in \mathbb{R}^{n}$, the functions $a_{i}(t): T \rightarrow \mathbb{R}, i=0, \ldots, n$, are defined in the compact index set $T$ in the form (6). Then problem $(P)$ takes the form

$$
\begin{gathered}
\min _{x} c(x), \\
\text { s.t. } f(x, t)=\sum_{i=1}^{n} a_{i}(t) x_{i}+a_{0}(t) \leq 0, \forall t \in T \subset \mathbb{R}^{s} .
\end{gathered}
$$

We assume that the functions $c(x), a_{i}(t), i=0,1, \ldots, n$, are sufficiently smooth.

The feasible set $X$ in (10) is as follows:

$$
X=\left\{x \in \mathbb{R}^{n}: \sum_{i=1}^{n} a_{i}(t) x_{i}+a_{0}(t) \leq 0, \forall t \in T\right\} .
$$

Let $T^{*}$ be the set of immobile indices w.r.t. the constraints of problem (10) (see (5)). Given $t \in T^{*}$, suppose that the sets $\bar{J}^{-}(t), \bar{J}^{+}(t), \bar{J}(t)$ are defined as in (8), and $\mathcal{M}(t)$ denotes the subspace generated by the vectors $e_{j}, j \in \bar{J}(t)$.

Proposition 3. Consider the convex SIP problem (10) with the feasible set X. Suppose that an immobile index $\bar{t} \in T^{*}$ satisfies the following condition:

$$
q(\bar{t}, l) \leq 1, \forall l \in L(\bar{t}) .
$$


Then there exists $\bar{x}=x(\bar{t}) \in X$ such that

$$
\begin{gathered}
\frac{\partial f^{T}(\bar{x}, \bar{t})}{\partial t} e_{j}<0, j \in \bar{J}^{-}(\bar{t}), \quad \frac{\partial f^{T}(\bar{x}, \bar{t})}{\partial t} e_{j}>0, j \in \bar{J}^{+}(\bar{t}), \frac{\partial f^{T}(\bar{x}, \bar{t})}{\partial t} e_{j}=0, j \in \bar{J}(\bar{t}) \\
l^{T} \frac{\partial^{2} f(\bar{x}, \bar{t})}{\partial t^{2}} l<0 \quad \forall l \in L(\bar{t}) \cap \mathcal{M}(\bar{t}) .
\end{gathered}
$$

Proof. Conditions (9) and (11) imply

$$
q(\bar{t}, l)=0, l \in L(\bar{t}) \backslash \mathcal{M}(\bar{t}) ; q(\bar{t}, l)=1, l \in L(\bar{t}) \cap \mathcal{M}(\bar{t}) .
$$

Denote by $(\hat{P})$ the following auxiliary SIP problem:

$$
\begin{aligned}
& \min _{\xi, x} \xi, \\
& \text { s.t. } \quad x \in X, l^{T} \frac{\partial^{2} f(x, \bar{t})}{\partial t^{2}} l \leq \xi, \forall l \in L(\bar{t}) \cap \mathcal{M}(\bar{t}) .
\end{aligned}
$$

In problem $(\hat{P})$, the set $X$ is convex, the objective function is convex, and the constraints are linear w.r.t. $x$. These constraints satisfy the condition $C Q 1$ :

$$
\exists(\bar{x}, \bar{\xi}), \bar{x} \in X, \bar{\xi}=\max _{\|l\|=1} l^{T} \frac{\partial^{2} f(\bar{x}, \bar{t})}{\partial t^{2}} l+1, \text { such that } l^{T} \frac{\partial^{2} f(\bar{x}, \bar{t})}{\partial t^{2}} l<\bar{\xi}, \forall l \in L(\bar{t}) \cap \mathcal{M}(\bar{t}) .
$$

Hence (see Proposition 2) they satisfy the condition $C Q 2$ as well. Consequently, by Proposition 1 there exist $n+1$ vectors $l_{i} \in L(\bar{t}) \cap \mathcal{M}(\bar{t}), i=1, \ldots, n+1$, such that $\operatorname{val}(\hat{P})=\operatorname{val}\left(\hat{P}_{D}\right)$ where problem $\left(\hat{P}_{D}\right)$ is as follows:

$$
\begin{aligned}
& \quad \min _{\xi, x} \xi \\
& \left(\hat{P}_{D}\right) \quad \text { s.t. } \quad x \in X, l_{i}^{T} \frac{\partial^{2} f(x, \bar{t})}{\partial t^{2}} l_{i} \leq \xi, \quad i=1, \ldots, n+1 .
\end{aligned}
$$

By (14) and Definition 2, for each vector $l_{i}, i=1, \ldots, n+1$, there exists $x^{(i)} \in X$ such that

$$
l_{i}^{T} \frac{\partial^{2} f\left(x^{(i)}, \bar{t}\right)}{\partial t^{2}} l_{i}<0, i=1, \ldots, n+1 .
$$

Since $\bar{t}$ is an immobile index and $x^{(i)}$ is a feasible solution to problem (10), then for any $k \in\{1, \ldots, n+1\}, k \neq i$ it holds $l_{k}^{T} \frac{\partial^{2} f\left(x^{(i)}, \bar{t}\right)}{\partial t^{2}} l_{k} \leq 0$. Consider the vector $\check{x}:=\sum_{k=1}^{n+1} \frac{x^{(k)}}{n+1}$. Evidently, $\check{x} \in X$. Having substituted this vector in the left-hand side of the constraints of the problem $\left(\hat{P}_{D}\right)$, we get

$$
l_{i}^{T} \frac{\partial^{2} f(\check{x}, \bar{t})}{\partial t^{2}} l_{i}=\sum_{k=1}^{n+1}\left(l_{i}^{T} \frac{\partial^{2} f\left(x^{(k)}, \bar{t}\right)}{\partial t^{2}} l_{i} /(n+1)\right)<0, \quad i=1, \ldots, n+1,
$$

and therefore conclude that $\operatorname{val}\left(\hat{P}_{D}\right)<0$. Hence $\operatorname{val}(\hat{P})<0$ that implies the existence of a vector $x^{*} \in X$ such that

$$
\frac{\partial f^{T}\left(x^{*}, \bar{t}\right)}{\partial t} l=0, l^{T} \frac{\partial^{2} f\left(x^{*}, \bar{t}\right)}{\partial t^{2}} l<0, \forall l \in L(\bar{t}) \cap \mathcal{M}(\bar{t}) .
$$


From (14), it follows that $q(\bar{t}, l)=0, l \in L(\bar{t}) \backslash \mathcal{M}(\bar{t})$. Hence by Definition 2, for each vector $e_{j}, j \in \bar{J}^{ \pm}(\bar{t})$, there exists $y^{(j)} \in X$ such that

$$
\frac{\partial f^{T}\left(y^{(j)}, \bar{t}\right)}{\partial t} e_{j}<0, j \in \bar{J}^{-}(\bar{t}), \frac{\partial f^{T}\left(y^{(j)}, \bar{t}\right)}{\partial t} e_{j}>0, j \in \bar{J}^{+}(\bar{t})
$$

Since $\bar{t}$ is an immobile index and $y^{(j)}$ is a feasible solution to problem (10), then for any $j \in \bar{J}^{ \pm}(\bar{t})$ the following conditions are satisfied:

$$
\begin{gathered}
\frac{\partial f^{T}\left(y^{(j)}, \bar{t}\right)}{\partial t} l=0, l^{T} \frac{\partial^{2} f\left(y^{(j)}, \bar{t}\right)}{\partial t^{2}} l \leq 0, l \in L(\bar{t}) \cap \mathcal{M}(\bar{t}) \\
\frac{\partial f^{T}\left(y^{(j)}, \bar{t}\right)}{\partial t} l \leq 0, l \in L(\bar{t}) \backslash \mathcal{M}(\bar{t}) .
\end{gathered}
$$

Consider $\bar{x}=\left(x^{*}+\sum_{j \in \bar{J}^{ \pm}(\bar{t})} y^{(j)}\right) / r, r=1+\left|\bar{J}^{-}(\bar{t})\right|+\left|\bar{J}^{+}(\bar{t})\right|$. It is evident that $\bar{x} \in X$. From (15)-(18), and from the linearity of $f(x, t)$ w.r.t. $x$ it follows that $\bar{x}$ satisfies conditions (12), (13). The proposition is proved.

Corollary 1. Conditions (12) and (13) imply that given an immobile index $\bar{t}$ satisfying (11), there exists $\bar{x}=x(\bar{t}) \in X$ such that

$$
\frac{\partial f^{T}(\bar{x}, \bar{t})}{\partial t} l=0, l^{T} \frac{\partial^{2} f(\bar{x}, \bar{t})}{\partial t^{2}} l<0, l \in L(\bar{t}) \cap \mathcal{M}(\bar{t}) ; \quad \frac{\partial f^{T}(\bar{x}, \bar{t})}{\partial t} l<0, l \in L(\bar{t}) \backslash \mathcal{M}(\bar{t}) .
$$

\subsection{Implicit optimality criterion}

Let us make the following assumption.

Assumption 1. All immobile indices in problem (10) satisfy condition (11), that means here $q(t, l) \leq 1, \forall l \in L(t), \forall t \in T^{*}$.

Assumption 1 is considered to be trivially fulfilled if $T^{*}=\emptyset$.

Lemma 1. Let Assumption 1 be fulfilled. Then the set of immobile indices in problem (10) is finite:

$$
T^{*}=\left\{t_{j}^{0}, j \in I^{*}\right\}, I^{*}=\left\{1, \ldots, p^{0}\right\}, \quad 0 \leq p^{0}<\infty .
$$

Here and in what follows we assume the set $\{1, \ldots, k\}$ to be empty if $k=0$.

Proof of the lemma is presented in the Appendix.

Notice that by Proposition 2 , if $T^{*} \neq \emptyset$ then the Slater type conditions I and II cannot be fulfilled. Nevertheless Assumption 1 can be satisfied in such situation. Therefore for the SIP problem considered here this assumption is less restrictive than the Slater type conditions.

For any feasible solution $x \in X$ of problem (10), denote by

$$
T_{a}(x)=\{t \in T: f(x, t)=0\}
$$

the set of active indices (active index set) at $x$. 
Proposition 4. Given the convex SIP problem in the form (10), let Assumption 1 be fulfilled. Then there exists a feasible solution $\tilde{x} \in X$ such that

$$
\begin{gathered}
T_{a}(\tilde{x})=T^{*}, \\
\frac{\partial f^{T}(\tilde{x}, t)}{\partial t} l=0, l^{T} \frac{\partial^{2} f(\tilde{x}, t)}{\partial t^{2}} l<0, l \in L(t) \cap \mathcal{M}(t), \\
\frac{\partial f^{T}(\tilde{x}, t)}{\partial t} l<0, l \in L(t) \backslash \mathcal{M}(t), \forall t \in T^{*} .
\end{gathered}
$$

Proof. From Assumption 1 and Corollary 1, it follows that there exist feasible solutions $x^{(j)} \in X, j \in I^{*}$, such that the following conditions take place

$$
\begin{gathered}
f\left(x^{(j)}, t_{j}^{0}\right)=0, \\
\frac{\partial f^{T}\left(x^{(j)}, t_{j}^{0}\right)}{\partial t} l=0, l^{T} \frac{\partial^{2} f\left(x^{(j)}, t_{j}^{0}\right)}{\partial t^{2}} l<0, l \in L\left(t_{j}^{0}\right) \cap \mathcal{M}\left(t_{j}^{0}\right), \\
\frac{\partial f^{T}\left(x^{(j)}, t_{j}^{0}\right)}{\partial t} l<0, l \in L\left(t_{j}^{0}\right) \backslash \mathcal{M}\left(t_{j}^{0}\right), \quad j \in I^{*} .
\end{gathered}
$$

For $j \in I^{*}$, the latest relations are sufficient conditions for point $t_{j}^{0}$ to be a strict local solution of the problem $\max _{\tau \in T} f\left(x^{(j)}, \tau\right)$. Hence there exists a number $\varepsilon>0$ such that

$$
f\left(x^{(j)}, t\right)<f\left(x^{(j)}, t_{j}^{0}\right)=0, t \in T_{\varepsilon}\left(t_{j}^{0}\right) \backslash t_{j}^{0}, j \in I^{*}
$$

where

$$
T_{\varepsilon}(t)=T \cap\left\{\tau \in \mathbb{R}^{s}:\|t-\tau\| \leq \varepsilon\right\} .
$$

Denote by $\tilde{T}$ the closure of the set $T \backslash \bigcup_{j \in I^{*}} T_{\varepsilon}\left(t_{j}^{0}\right)$ and put $\tilde{Q}=X$. It is evident that the set $\tilde{T}$ is compact and the set $\tilde{Q}$ is convex.

Consider the following constraints:

$$
x \in \tilde{Q}, f(x, t) \leq 0, \forall t \in \tilde{T} .
$$

By construction, the set of immobile indices w.r.t. these constraints is empty, i.e.

$$
\tilde{T}^{*}=\{t \in \tilde{T}: f(x, t)=0, \forall x \in \tilde{X}\}=\emptyset,
$$

where $\tilde{X}=\left\{x \in \mathbb{R}^{n}: x \in \tilde{Q}, f(x, t) \leq 0, \forall t \in \tilde{T}\right\}$. Consequently, by Proposition 2, constraints (26) satisfy the Slater type condition I, i.e. there exists $y \in \tilde{Q}=X$ such that

$$
f(y, t)<0, \forall t \in \tilde{T} .
$$

Observe that the conditions $y \in X$ and $t_{j}^{0} \in T^{*}, j \in I^{*}$, imply

$$
\begin{gathered}
\frac{\partial f^{T}\left(y, t_{j}^{0}\right)}{\partial t} l=0, l^{T} \frac{\partial^{2} f\left(y, t_{j}^{0}\right)}{\partial t^{2}} l \leq 0, l \in L\left(t_{j}^{0}\right) \cap \mathcal{M}\left(t_{j}^{0}\right) \\
\frac{\partial f^{T}\left(y, t_{j}^{0}\right)}{\partial t} l \leq 0, l \in L\left(t_{j}^{0}\right) \backslash \mathcal{M}\left(t_{j}^{0}\right) .
\end{gathered}
$$


Let us consider the vector $\tilde{x}=\left(y+\sum_{j \in I^{*}} x^{(j)}\right) /\left(\left|I^{*}\right|+1\right)$. From conditions $y \in X, x^{(j)} \in X, j \in I^{*}$, and (23), (24), (27), (28), taking into account the convexity of the set $X$ and the linearity of function $f(x, t)$ w.r.t. $x$, we conclude that the vector $\tilde{x}$ constructed above belongs to $X$ and satisfies (20)-(22). The proposition is proved.

To simplify notation, let us denote

$$
\begin{aligned}
& \bar{J}_{j}^{ \pm}:=\bar{J}^{ \pm}\left(t_{j}^{0}\right), \bar{J}_{j}:=\bar{J}\left(t_{j}^{0}\right), s_{j}:=\left|\bar{J}_{j}\right|, r_{j}=s-s_{j}, \bar{J}_{j}^{*}=\bar{J}_{j} \cap\left(J^{+}\left(t_{j}^{0}\right) \cup J^{-}\left(t_{j}^{0}\right)\right) \\
& M_{j}=\left(e_{i}, i \in \bar{J}_{j} \backslash J^{+}\left(t_{j}^{0}\right),-e_{i}, i \in \bar{J}_{j} \cap J^{+}\left(t_{j}^{0}\right)\right) \in \mathbb{R}^{n \times s_{j}} \\
& N_{j}=\left(e_{i}, i \in \bar{J}_{j}^{-},-e_{i}, i \in \bar{J}_{j}^{+}\right) \in \mathbb{R}^{s \times r_{j}}, j \in I^{*}
\end{aligned}
$$

The following theorem holds.

Theorem 1. [Implicit optimality criterion] Suppose that Assumption 1 is satisfied for the convex problem (10). Then a feasible solution $x^{0} \in X$ is optimal to this problem if and only if there exists a set of indices

$$
\left\{t_{j}, j \in I\right\} \subset T_{a}\left(x^{0}\right) \backslash T^{*},|I| \leq n,
$$

such that the vector $x^{0}$ is optimal to the following auxiliary problem:

$$
\begin{array}{ll} 
& \min _{x} c(x), \\
\text { s.t. } & f\left(x, t_{j}^{0}\right)=0, \frac{\partial f^{T}\left(x, t_{j}^{0}\right)}{\partial t} N_{j} \leq 0, \frac{\partial f^{T}\left(x, t_{j}^{0}\right)}{\partial t} M_{j}=0, \\
& \beta_{j}^{T} M_{j}^{T} \frac{\partial^{2} f\left(x, t_{j}^{0}\right)}{\partial t^{2}} M_{j} \beta_{j} \leq 0, \forall \beta_{j}=\left(\beta_{j i}, i \in \bar{J}_{j}\right), \beta_{j i} \geq 0, i \in \bar{J}_{j}^{*}, j \in I^{*} ; \\
& f\left(x, t_{j}\right) \leq 0, j \in I .
\end{array}
$$

Proof. Sufficiency. Suppose that vector $x^{0} \in X$ is an optimal solution of problem (31) with some index set (30). Denote by $Y$ the feasible set of problem (31). Since $X \subset Y$, then $x^{0}$ is optimal in problem (10) as well.

Necessity. For $\varepsilon>0$, denote $T(\varepsilon)=T \backslash \operatorname{int}\left(\bigcup_{j \in I^{*}} T_{\varepsilon}\left(t_{j}^{0}\right)\right)$, the set $T_{\varepsilon}(t)$ being defined in (25). Consider the family of problems

$$
\begin{gathered}
(P(\varepsilon)) \quad \min _{x} c(x), \\
\text { s.t. } x \in \bar{Y} \cap B, \quad f(x, t) \leq 0, \forall t \in T(\varepsilon),
\end{gathered}
$$

where $B=B\left(\varepsilon_{0}, x^{0}\right)=\left\{x \in \mathbb{R}^{n}:\left\|x-x^{0}\right\| \leq \varepsilon_{0}\right\}, \varepsilon_{0}>\left\|\tilde{x}-x^{0}\right\|$ is any fixed number, $\tilde{x}$ is a feasible solution of problem (10) satisfying (20)-(22), $x^{0}$ is an optimal solution to problem (10), and

$$
\begin{aligned}
& \bar{Y}:=\quad\left\{x \in \mathbb{R}^{n}: f\left(x, t_{j}^{0}\right)=0, \frac{\partial f^{T}\left(x, t_{j}^{0}\right)}{\partial t} N_{j} \leq 0, \frac{\partial f^{T}\left(x, t_{j}^{0}\right)}{\partial t} M_{j}=0,\right. \\
& \left.\beta_{j}^{T} M_{j}^{T} \frac{\partial^{2} f\left(x, t_{j}^{0}\right)}{\partial t^{2}} M_{j} \beta_{j} \leq 0, \forall \beta_{j}=\left(\beta_{j i}, i \in \bar{J}_{j}\right), \beta_{j i} \geq 0, i \in \bar{J}_{j}^{*}, j \in I^{*}\right\} .
\end{aligned}
$$

Notice here that the sets $\bar{Y}, B$, and hence, $\bar{Y} \cap B$ are convex. 
Let $y(\varepsilon)$ be an optimal solution to problem $P(\varepsilon)$. It is evident that

$$
c(y(\varepsilon)) \leq c\left(x^{0}\right)
$$

The constraints of problem $(P(\varepsilon))$ satisfy the Slater type condition I:

$$
\tilde{x} \in \bar{Y} \cap B \text { and } f(\tilde{x}, t)<0, \forall t \in T(\varepsilon) \text {, }
$$

where $\tilde{x}$ is a feasible solution of problem (10) satisfying (20)-(22). Hence by Propositions 1 and 2 , there exists a set of indices $t_{i}(\varepsilon) \in T(\varepsilon), i=1, \ldots, n$, such that

$$
\operatorname{val}(P(\varepsilon))=\operatorname{val}\left(P_{D}(\varepsilon)\right),
$$

where $\left(P_{D}(\varepsilon)\right)$ is the following NLP problem:

$$
\begin{gathered}
\left(P_{D}(\varepsilon)\right) \quad \min _{x} c(x), \\
\text { s.t. } x \in \bar{Y} \cap B, \quad f\left(x, t_{i}(\varepsilon)\right) \leq 0, i=1, \ldots, n .
\end{gathered}
$$

Since $t_{i}(\varepsilon) \in T, i=1, \ldots, n$, for all $\varepsilon>0$, there exists a sequence $\left\{\varepsilon_{j}\right\}: \varepsilon_{j} \rightarrow+0$ as $j \rightarrow \infty$, such that

$$
t_{i}\left(\varepsilon_{j}\right) \rightarrow \bar{t}_{i} \in T \text { as } j \rightarrow \infty, i=1, \ldots, n .
$$

Consider the NLP problem

$$
\begin{gathered}
\min _{x} c(x), \\
\text { s.t. } x \in \bar{Y} \cap B, \quad f\left(x, \bar{t}_{i}\right) \leq 0, i=1, \ldots, n .
\end{gathered}
$$

This problem has an optimal solution. Let $\bar{y}$ be optimal in $(\bar{P})$. It is evident that $c(\bar{y}) \leq c\left(x^{0}\right)$. Let us show that $c(\bar{y})=c\left(x^{0}\right)$. Suppose that $c(\bar{y})<c\left(x^{0}\right)$ and consider the vector

$$
x^{*}(\lambda)=(1-\lambda) \bar{y}+\lambda \tilde{x} .
$$

By construction $\tilde{x} \in \bar{Y} \cap B$ and $\bar{y} \in \bar{Y} \cap B$. Hence

$$
x^{*}(\lambda) \in \bar{Y} \cap B \text { for all } \lambda \in[0,1] .
$$

Denote $\bar{I}^{*}=\left\{i \in\{1, \ldots, n\}: \bar{t}_{i} \in T^{*}\right\}, \bar{I}_{0}^{*}=\{1, \ldots, n\} \backslash \bar{I}^{*}$. According to Proposition 6 (see Appendix), for any $\lambda \in(0,1]$ there exist numbers $\Delta_{i}(\lambda)>0, i \in \bar{I}^{*}$, such that

$$
f\left(x^{*}(\lambda), t\right) \leq 0, t \in T_{\Delta_{i}(\lambda)}\left(\bar{t}_{i}\right), i \in \bar{I}^{*} .
$$

For $t=\bar{t}_{i}+\Delta t l, l \in L\left(\bar{t}_{i}\right), i \in \bar{I}_{0}^{*}, \Delta t \geq 0$, one has

$$
\begin{gathered}
f\left(x^{*}(\lambda), t\right) \leq(1-\lambda) f(\bar{y}, t)+\lambda f(\tilde{x}, t)= \\
(1-\lambda)\left[f\left(\bar{y}, \bar{t}_{i}\right)+\Delta t \frac{\partial f^{T}\left(\bar{y}_{,} \bar{t}_{i}\right) l}{\partial t}+o(\Delta t)\right]+\lambda\left[f\left(\tilde{x}, \bar{t}_{i}\right)+\Delta t \frac{\partial f^{T}\left(\tilde{x}, \bar{t}_{i}\right) l}{\partial t}+o(\Delta t)\right] .
\end{gathered}
$$

Taking into account that by construction $f\left(\bar{y}, \bar{t}_{i}\right) \leq 0$ and $f\left(\tilde{x}, \bar{t}_{i}\right)<0, i \in \bar{I}_{0}^{*}$, we conclude that for any $\lambda \in(0,1]$ there exist numbers $\Delta_{i}(\lambda)>0, i \in \bar{I}_{0}^{*}$, such that

$$
f\left(x^{*}(\lambda), t\right) \leq 0, t \in T_{\Delta_{i}(\lambda)}\left(\bar{t}_{i}\right), i \in \bar{I}_{0}^{*} .
$$


Let us put $\bar{\lambda}=\left(c\left(x^{0}\right)-c(\bar{y})\right) /(2(c(\tilde{x})-c(\bar{y})))$. Taking into account that by assumption $c(\bar{y})<$ $c\left(x^{0}\right)$ and by construction $c\left(x^{0}\right) \leq c(\tilde{x}), c(\bar{y}) \leq c(\tilde{x})$, we conclude that $\bar{\lambda} \in(0,0.5]$.

Notice that

$$
c\left(x^{*}(\bar{\lambda})\right) \leq c\left(x^{0}\right)-\frac{1}{2}\left(c\left(x^{0}\right)-c(\bar{y})\right) .
$$

It follows from (36) and (37) that for $\bar{\lambda}$ there exists a number $\bar{\Delta}=\Delta(\bar{\lambda})>0$ such that

$$
f\left(x^{*}(\bar{\lambda}), t\right) \leq 0, t \in T_{\bar{\Delta}}\left(\bar{t}_{i}\right), i=1, \ldots, n .
$$

According to (34) there exists a number $\bar{k} \in \mathbb{N}$ such that

$$
t_{i}\left(\varepsilon_{j}\right) \in T_{\bar{\Delta}}\left(\bar{t}_{i}\right), i=1, \ldots, n, \forall j \geq \bar{k} .
$$

Relations (35), (39) and (40) imply the feasibility of the vector $x^{*}(\bar{\lambda})$ in all problems $\left(P_{D}\left(\varepsilon_{j}\right)\right)$ with $j \geq \bar{k}$. Consequently

$$
c\left(x^{*}(\bar{\lambda})\right) \geq c\left(y\left(\varepsilon_{j}\right)\right), \forall j \geq \bar{k} .
$$

From (38) and (41) we get $c\left(x^{0}\right)-\left(c\left(x^{0}\right)-c(\bar{y})\right) / 2 \geq c\left(y\left(\varepsilon_{j}\right)\right), \forall j \geq \bar{k}$. Let us pass on to the limit as $j \rightarrow \infty$ in the latest inequality, taking into account that by Corollary 3 (see Appendix) it holds $\lim _{\varepsilon \rightarrow+0} c(y(\varepsilon))=c\left(x^{0}\right)$. As a result we get

$$
c\left(x^{0}\right)-\left(c\left(x^{0}\right)-c(\bar{y})\right) / 2 \geq c\left(x^{0}\right)
$$

that contradicts the assumption $c(\bar{y})<c\left(x^{0}\right)$. Therefore $c(\bar{y})=c\left(x^{0}\right)$ and the vector $x^{0}$ is an optimal solution to problem $(\bar{P})$. Note that by construction $f\left(x, \bar{t}_{i}\right)=0, i \in \bar{I}^{*}$, for all $x \in \bar{Y}$. Hence if $x^{0}$ is optimal in problem $(\bar{P})$ then it is optimal in problem (31) with $\left\{t_{j}, j \in I\right\}=\left\{\bar{t}_{i}, i \in \bar{I}_{0}^{*}\right\}$. The theorem is proved.

\section{Explicit optimality conditions}

\subsection{Necessary and sufficient optimality conditions}

The implicit optimality criterion proved in the previous section allows to replace the check of optimality of a feasible solution $x^{0}$ in the SIP problem (10) by the check of the optimality of $x^{0}$ in the auxiliary problem (31). In the next part of the paper, we will study optimality conditions for the auxiliary problem (31) and obtain new explicit optimality conditions for the original SIP problem. These conditions will be compared with some known optimality results in SIP.

Notice that problem (31) is a SIP problem that is linear w.r.t. decision variable $x$ and is quadratic w.r.t. index variables $\beta_{j} \in \mathbb{R}^{s_{j}}, j \in I^{*}$. Below we will show that this problem can be written in the form of the problem that can be treated in a similar way as problems of Semidefinite Programming (SDP).

Let us introduce the following notation:

$$
\begin{gathered}
Q(I):=\left\{x \in \mathbb{R}^{n}: f\left(x, t_{j}\right) \leq 0, j \in I, f\left(x, t_{j}^{0}\right)=0, \frac{\partial f^{T}\left(x, t_{j}^{0}\right)}{\partial t} N_{j} \leq 0, \frac{\partial f^{T}\left(x, t_{j}^{0}\right)}{\partial t} M_{j}=0, j \in I^{*}\right\}, \\
A_{i j}:=\frac{\partial^{2} a_{i}\left(t_{j}^{0}\right)}{\partial t^{2}}, i=0,1, \ldots, n, \mathcal{A}_{j}(x):=\sum_{i=1}^{n} A_{i j} x_{i}+A_{0 j}=\frac{\partial^{2} f\left(x, t_{j}^{0}\right)}{\partial t^{2}}, j \in I^{*} .
\end{gathered}
$$


It is evident that $Q(I)$ is a polyhedron in $\mathbb{R}^{n}$. Using the notations introduced above, we can rewrite problem (31) in the equivalent form

$$
\begin{aligned}
& \min _{x} c(x), \\
\text { s.t. } & x \in Q(I), \\
& \beta_{j}^{T} M_{j}^{T} \mathcal{A}_{j}(x) M_{j} \beta_{j} \leq 0, \forall \beta_{j}=\left(\beta_{j i}, i \in \bar{J}_{j}\right), \beta_{j i} \geq 0, i \in \bar{J}_{j}^{*},\left\|\beta_{j}\right\|=1, j \in I^{*} .
\end{aligned}
$$

If Assumption 1 is satisfied for the original SIP problem (10), then

$$
\exists \tilde{x} \in Q(I): \beta_{j}^{T} M_{j}^{T} \mathcal{A}_{j}(\tilde{x}) M_{j} \beta_{j}<0, \forall \beta_{j}=\left(\beta_{j i}, i \in \bar{J}_{j}\right), \beta_{j i} \geq 0, i \in \bar{J}_{j}^{*},\left\|\beta_{j}\right\|=1, j \in I^{*} ;
$$

i.e, the constraints of problem (42) satisfy the Slater condition ([3]). The following optimality criterion can be formulated in this case.

Theorem 2. Suppose that condition (43) is satisfied. Then a feasible solution $x^{0}$ of problem (42) is optimal if and only if there exist a set of numbers and vectors

$$
\alpha_{j} \geq 0, j \in I ; \lambda_{j} \in \mathbb{R}, \gamma_{j}=\left(\gamma_{j 1}, \ldots, \gamma_{j s}\right)^{T} \in \mathbb{R}^{s}, \gamma_{j i} \geq 0, i \in \bar{J}_{j}^{-}, \gamma_{j i} \leq 0, i \in \bar{J}_{j}^{+}, j \in I^{*},
$$

and a set of vectors

$$
\begin{aligned}
& \beta_{j}(k) \in L_{a}^{j}\left(x^{0}\right):=\left\{\beta=\left(\beta_{i}, i \in \bar{J}_{j}\right) \neq 0, \beta^{T} M_{j}^{T} \mathcal{A}_{j}\left(x^{0}\right) M_{j} \beta=0, \beta_{i} \geq 0, i \in \bar{J}_{j}^{*}\right\}, \\
& k=1, \ldots, \kappa_{j}, s_{j} \geq \kappa_{j} \geq 0, j \in I^{*}, \sum_{j \in I^{*}} \kappa_{j} \leq n,
\end{aligned}
$$

such that the following equalities hold:

$$
\begin{gathered}
\frac{\partial c\left(x^{0}\right)}{\partial x_{i}}+\sum_{j \in I^{*}}\left(a_{i}\left(t_{j}^{0}\right) \lambda_{j}+\gamma_{j}^{T} \frac{\partial a_{i}\left(t_{j}^{0}\right)}{\partial t}+\sum_{k=1}^{\kappa_{j}} \beta_{j}^{T}(k) M_{j}^{T} A_{i j} M_{j} \beta_{j}(k)\right)+\sum_{j \in I} \alpha_{j} a_{i}\left(t_{j}\right)=0, i=1, \ldots, n, \\
\gamma_{j}^{T} \frac{\partial f\left(x^{0}, t_{j}^{0}\right)}{\partial t}=0, j \in I^{*} .
\end{gathered}
$$

Given a symmetric matrix $A$, let us write $A \prec 0$ if $A$ is negative definite and $A \preceq 0$ if $A$ is negative semi-definite.

Problem (42) can be consider as a slight generalization of the following SDP problem:

$$
\begin{gathered}
\min _{x} c(x), \\
\text { s.t. } x \in Q(I), M_{j}^{T} \mathcal{A}_{j}(x) M_{j} \preceq 0, j \in I^{*},
\end{gathered}
$$

and Theorem 2 can be reformulated in terms of SDP as follows.

Theorem 3. Suppose that condition (43) is satisfied. Then a feasible solution $x^{0}$ is optimal to problem (42) if and only if there exist a set of numbers and vectors (44) and a set of positive semidefinite matrices $\Omega_{j}=B_{j} B_{j}^{T}$ with $B_{j}=\left(\beta_{j}(k), k=1, \ldots, \kappa_{j}\right)$, where $\beta_{j}(k)=\left(\beta_{j i}(k), i \in\right.$ $\left.\bar{J}_{j}\right), \beta_{j i}(k) \geq 0, i \in \bar{J}_{j}^{*}, k=1, \ldots, \kappa_{j}, j \in I^{*}$, such that

$$
\begin{aligned}
& \frac{\partial c\left(x^{0}\right)}{\partial x_{i}}+\sum_{j \in I^{*}}\left(a_{i}\left(t_{j}^{0}\right) \lambda_{j}+\gamma_{j}^{T} \frac{\partial a_{i}\left(t_{j}^{0}\right)}{\partial t}+\Omega_{j} \bullet M_{j}^{T} A_{i j} M_{j}\right)+\sum_{j \in I} \alpha_{j} a_{i}\left(t_{j}\right)=0, i=1, \ldots, n, \\
& \gamma_{j}^{T} \frac{\partial f\left(x^{0}, t_{j}^{0}\right)}{\partial t}=0, \Omega_{j} \bullet M_{j}^{T} \mathcal{A}\left(x^{0}\right) M_{j}=0, j \in I^{*} .
\end{aligned}
$$


Here the symbol "•" stays for the trace inner product defined in the space $\mathcal{S}(k)$ of $k \times k$ symmetric matrices: $A \bullet B:=\operatorname{trace}(A B)$ for $A, B \in \mathcal{S}(k)$.

It is easy to see that Theorem 3 is a simple generalization of the well-known result on SDP theory (see, for example, [3]). For other optimality conditions for SDP see [21], [24] et al.

The next theorem is an immediate consequence of Theorems 1 and 2 .

Theorem 4. [Explicit optimality criterion] Let Assumption 1 be fulfilled for the convex SIP problem (10). Then a feasible solution $x^{0} \in X$ is optimal to this problem if and only if there exist a set of indices (30), a set of vectors (45), and a set of numbers and vectors (44) such that relations (46) are true.

Corollary 2. Let Assumption 1 be fulfilled. Then a feasible solution $x^{0} \in X$ is optimal to SIP problem (10) if and only if there exist sets of indices (30) and vectors (45) such that vector $x^{0}$ is optimal in the following Convex Programming (CP) problem with linear constraints:

$$
\begin{gathered}
\min _{x} c(x), \\
\text { s.t. } x \in Q(I), \beta_{j}^{T}(k) M_{j}^{T}\left(\sum_{i=1}^{n} A_{i j} x_{i}+A_{0 j}\right) M_{j} \beta_{j}(k) \leq 0, k=1, \ldots, \kappa_{j}, j \in I^{*} .
\end{gathered}
$$

Corollary 2 permits to replace the check of optimality of some feasible solution in the SIP problem (10) by the check of its optimality in the auxiliary CP problem (49).

Notice that equalities (46) can be rewritten in the form

$$
\begin{aligned}
& \frac{\partial c\left(x^{0}\right)}{\partial x}+\sum_{j \in I^{*}}\left(\lambda_{j} \frac{\partial f\left(x^{0}, t_{j}^{0}\right)}{\partial x}+\frac{\partial^{2} f\left(x^{0}, t_{j}^{0}\right)}{\partial x \partial t} \gamma_{j}+\sum_{k=1}^{\kappa_{j}} \frac{\partial}{\partial x}\left(\beta_{j}^{T}(k) M_{j}^{T} \frac{\partial^{2} f\left(x^{0}, t_{j}^{0}\right)}{\partial t^{2}} M_{j} \beta_{j}(k)\right)\right) \\
& +\sum_{j \in I} \alpha_{j} \frac{\partial f\left(x^{0}, t_{j}\right)}{\partial x}=0, \quad \gamma_{j}^{T} \frac{\partial f\left(x^{0}, t_{j}^{0}\right)}{\partial t}=0, j \in I^{*} .
\end{aligned}
$$

Remark 1. If the constraints of the convex problem (10) satisfy the condition CQ1 or CQ2, then by Proposition $2, T^{*}=\emptyset$. Therefore $I^{*}=\emptyset$ and conditions (50) coincide with the classical first order optimality conditions (KKT): $0=\frac{\partial c\left(x^{0}\right)}{\partial x}+\sum_{j \in I} \alpha_{j} \frac{\partial f\left(x^{0}, t_{j}\right)}{\partial x}$.

Remark 2. Consider the convex SIP problem (10). If $\frac{\partial^{2} f\left(x^{0}, t_{j}^{0}\right)}{\partial t^{2}} \prec 0, j \in I^{*}$, then $L_{a}^{j}\left(x^{0}\right)=$ $\emptyset, \forall j \in I^{*}$, (see (45)), and a feasible $x^{0} \in X$ is optimal in (10) if and only if it is optimal in the following CP problem:

$$
\min c(x) \text {, s.t. } x \in Q(I) \text {. }
$$

In this case the optimality conditions (50) take the form

$$
\frac{\partial c\left(x^{0}\right)}{\partial x}+\sum_{j \in I^{*}}\left(\lambda_{j} \frac{\partial f\left(x^{0}, t_{j}^{0}\right)}{\partial x}+\frac{\partial^{2} f\left(x^{0}, t_{j}^{0}\right)}{\partial x \partial t} \gamma_{j}\right)+\sum_{j \in I} \alpha_{j} \frac{\partial f\left(x^{0}, t_{j}\right)}{\partial x}=0, \gamma_{j}^{T} \frac{\partial f\left(x^{0}, t_{j}^{0}\right)}{\partial t}=0, j \in I^{*} .
$$


It should be noticed here that the results of this paper are also valid for convex problems with constraints in the form

$$
f_{i}\left(x, t^{(i)}\right) \leq 0, \forall t^{(i)} \in T_{i} \subset \mathbb{R}^{s_{i}}, i=1, \ldots p_{*},
$$

where $p_{*} \geq 1$ is a finite number, the sets $T_{i}, i=1, \ldots, p_{*}$, are bounded boxes in $\mathbb{R}^{s_{i}}$, functions $f_{i}\left(x, t^{(i)}\right), i=1, \ldots p_{*}$, are linear w.r.t. $x$.

Remark 3. The explicit optimality conditions for the convex SIP problem (10) were obtained here under Assumption 1 that is less restrictive than the Slater type conditions. In what follows we will show that when this assumption is removed, Theorem 4 continues to be true in its sufficient part, whereas the necessary conditions need an additional study.

\subsection{Example}

The optimality conditions for SIP problems satisfying the Slater type CQs, are well known (see [13], [14] et al.). In this paper, we have proved new implicit and explicit optimality conditions for convex SIP problems whose constraint functions are linear w.r.t. $x$. These conditions were formulated under Assumption 1 that is less restrictive than CQs mentioned above. Hence Theorems 1 and 4 proved in the paper give the more general optimality conditions. To illustrate this conclusion, we will consider here a simple example of a convex SIP problem with linear constraints in the form (51). Given a feasible solution that does not satisfy the known sufficient optimality conditions (we use here the results from [13], [14]), we will show that nevertheless this solution is optimal since it satisfies the optimality criterion given in Theorem 4 .

Let $x=\left(x_{1}, x_{2}, x_{3}, x_{4}\right)^{T} \in \mathbb{R}^{4}, t=\left(t_{1}, t_{2}\right)^{T} \in \mathbb{R}^{2}$, and

$$
\begin{gathered}
f_{1}(x, t)=-t_{1}^{2} x_{1}+t_{1} t_{2} x_{1}+t_{1} x_{2}+\left(\sin t_{1}\right) x_{3}+t_{1} x_{4}-t_{2}^{2}, \\
f_{2}(x, t)=t_{2} x_{1}+\left(t_{2}+1\right)^{2} x_{2}+\left(1-t_{2}\right) x_{3}+x_{4}-\left(t_{1}-3\right)^{2}+\left(t_{1}-3\right) t_{2} ; \\
T_{1}=\left\{t \in \mathbb{R}^{2}:-1 \leq t_{1} \leq 2,-1 \leq t_{2} \leq 2\right\}, T_{2}=\left\{t \in \mathbb{R}^{2}: 2 \leq t_{1} \leq 5,-1 \leq t_{2} \leq 2\right\} .
\end{gathered}
$$

Consider the following SIP problem:

$$
\begin{aligned}
& \min \left(x_{1}+x_{2}\right), \\
\text { s.t. } & f_{1}(x, t) \leq 0, \forall t \in T_{1}, \quad f_{2}(x, t) \leq 0, \forall t \in T_{2} .
\end{aligned}
$$

This problem admits a feasible solution $x^{0}=\left(x_{1}^{0}, x_{2}^{0}, x_{3}^{0}, x_{4}^{0}\right)^{T}$ such that

$$
\begin{gathered}
x_{1}^{0}=\frac{\sin 2+2}{2} \approx 1.455, \quad x_{2}^{0}=\frac{\left(x_{1}^{0}\right)^{2}+x_{1}^{0}(\sin 2-6)}{-2(\sin 2-2)} \approx-2.425, \\
x_{3}^{0}=x_{1}^{0}+2 x_{2}^{0}, \quad x_{4}^{0}=-3 x_{2}^{0}-x_{1}^{0} .
\end{gathered}
$$

Denote $t^{(1)}:=(0,0)^{T} \in T_{1}, t^{(2)}:=(3,0)^{T} \in T_{2}, t^{(3)}:=\left(2, x_{1}^{0}\right)^{T} \in T_{1}$. It is easy to verify that the indices $t^{(1)}, t^{(2)}$, and $t^{(3)}$ form the active index set at $x^{0}$, i.e. $f_{1}\left(x^{0}, t^{(1)}\right)=f_{1}\left(x^{0}, t^{(3)}\right)=$ $f_{2}\left(x^{0}, t^{(2)}\right)=0$.

There are two immobile indices in problem (52): $t^{(1)}$ and $t^{(2)}$. Calculate

$$
\frac{\partial^{2} f_{1}(x, t)}{\partial t_{1}^{2}}=-2 x_{1}-\sin \left(t_{1}\right) x_{3}, \quad \frac{\partial^{2} f_{1}(x, t)}{\partial t_{1} \partial t_{2}}=x_{1}, \quad \frac{\partial^{2} f_{1}(x, t)}{\partial t_{2}^{2}}=-2 .
$$


Then, evidently

$$
\frac{\partial^{2} f_{1}\left(x^{0}, t^{(1)}\right)}{\partial t^{2}}=\left(\begin{array}{cc}
-2 x_{1}^{0} & x_{1}^{0} \\
x_{1}^{0} & -2
\end{array}\right) \prec 0
$$

Since

$$
\begin{gathered}
\frac{\partial f_{2}(x, t)}{\partial t_{1}}=-2\left(t_{1}-3\right)+t_{2}, \quad \frac{\partial f_{2}(x, t)}{\partial t_{2}}=x_{1}+2\left(t_{2}+1\right) x_{2}-x_{3}+\left(t_{1}-3\right), \\
\frac{\partial^{2} f_{2}(x, t)}{\partial t_{1}^{2}}=-2, \quad \frac{\partial^{2} f_{2}(x, t)}{\partial t_{1} \partial t_{2}}=1, \quad \frac{\partial^{2} f_{2}(x, t)}{\partial t_{2}^{2}}=2 x_{2},
\end{gathered}
$$

then

$$
\frac{\partial^{2} f_{2}\left(x^{0}, t^{(2)}\right)}{\partial t^{2}}=\left(\begin{array}{cc}
-2 & 1 \\
1 & 2 x_{2}^{0}
\end{array}\right) \prec 0 .
$$

From the calculations provided above, we conclude that both immobile indices satisfy condition (11), and the sets $L_{a}^{1}\left(x^{0}\right)$ and $L_{a}^{2}\left(x^{0}\right)$ defined in (45) are empty. According to the explicit optimality criterion (Theorem 4), $x^{0}$ is optimal in problem (52) if and only if there exist real numbers and vectors $\lambda_{i}, \gamma_{i}=\left(\gamma_{i 1}, \gamma_{i 2}\right)^{T}, i=1,2 ; \alpha_{1} \geq 0$ such that

$$
\frac{\partial c\left(x^{0}\right)}{\partial x}+\sum_{i=1}^{2}\left(\lambda_{i} \frac{\partial f_{i}\left(x^{0}, t^{(i)}\right)}{\partial x}+\frac{\partial^{2} f_{i}\left(x^{0}, t^{(i)}\right)}{\partial x \partial t_{1}} \gamma_{i 1}+\frac{\partial^{2} f_{i}\left(x^{0}, t^{(i)}\right)}{\partial x \partial t_{2}} \gamma_{i 2}\right)+\alpha_{1} \frac{\partial f_{2}\left(x^{0}, t^{(3)}\right)}{\partial x}=0
$$

or

$$
\left(\begin{array}{l}
1 \\
1 \\
0 \\
0
\end{array}\right)+\lambda_{1}\left(\begin{array}{l}
0 \\
0 \\
0 \\
0
\end{array}\right)+\lambda_{2}\left(\begin{array}{l}
0 \\
1 \\
1 \\
1
\end{array}\right)+\gamma_{11}\left(\begin{array}{l}
0 \\
1 \\
1 \\
1
\end{array}\right)+\gamma_{12}\left(\begin{array}{l}
0 \\
0 \\
0 \\
0
\end{array}\right)+\gamma_{21}\left(\begin{array}{l}
0 \\
0 \\
0 \\
0
\end{array}\right)+\gamma_{22}\left(\begin{array}{c}
1 \\
2 \\
-1 \\
0
\end{array}\right)+\alpha_{1}\left(\begin{array}{c}
-4+2 x_{1}^{0} \\
2 \\
\sin 2 \\
2
\end{array}\right)=0 .
$$

Since the latest system has a solution

$$
\lambda_{1}=0, \lambda_{2}=\frac{1}{2 x_{1}^{0}-4} \approx-0.917, \gamma_{11}=\gamma_{12}=\gamma_{21}=0, \gamma_{22}=-\frac{1}{2}, \alpha_{1}=-\frac{1}{2\left(2 x_{1}^{0}-4\right)} \approx 0.458
$$

we conclude that the conditions of Theorem 4 are satisfied and $x^{0}$ is optimal in the SIP problem (52).

Finally, notice that the classical sufficient optimality conditions from [13, 14] are not satisfied for the given $x^{0}$ in problem (52).

\subsection{Optimality conditions for SIP problems without additional con- ditions}

It was shown above that under Assumption 1 that is less restrictive than the Slater type conditions, optimality conditions for the convex SIP problem (10) can be formulated in terms of optimality conditions for the auxiliary SDP-type problem (42). The latest problem satisfies the Slater condition and the optimality conditions for it can be written explicitly.

Relations (46), (48), and (50) continue to be sufficient (explicit) optimality conditions for problem (10) if Assumption 1 is violated. Notice that in this case, the set $T^{*}$ may consist of infinite number of elements. To take into account this situation, we can formulate sufficient optimality conditions as follows: 
Let $x^{0} \in X$ be a feasible solution of the convex SIP problem (10). Suppose that there exist a finite subset $\left\{t_{j}^{0}, j \in I^{*}\right\} \subset T^{*}, \quad\left|I^{*}\right|<\infty$, and a set (30) such that $x^{0}$ is optimal in problem (31) (or equivalently, in problem (42)), then it is optimal in (10) as well.

Notice that if Assumption 1 is violated, then problem (42) does not satisfy the Slater condition (43) and Theorems 3 and 4 do not hold in their necessary parts. In such situation, to check whether $x^{0} \in X$ is optimal in problem (42), one can use generalizations of the CQ-free optimality conditions (necessary and/or sufficient) used in SDP. Notice here that the sufficient parts of explicit optimality conditions derived in subsection 4.1, can be strengthen if we use for the auxiliary SDP-type problem (42) optimality conditions which require no CQs or other additional assumptions. For this purpose the detailed study of SDP-type problems without CQ has to be carried out. More details can be found in [10,24], some other papers, and in the forthcoming paper of the authors [21].

\section{Comparison with other known optimality conditions and conclusions}

In subsection 4.2 , we have considered an example of SIP problem that does not satisfy the Slater type conditions, but satisfies Assumption 1. We have showed that for this problem the classical optimality conditions are not satisfied while the optimality criterion proved in the paper (Theorem 4) holds.

In the present section, we will provide a more generalized comparison of the optimality conditions proved in this paper with other known results.

It was mentioned above that without Assumption 1, Theorem 4 continues to be true in its sufficient part. Now we will show that the conditions of this theorem describe a subset of optimal solutions that is larger than subsets of optimal solutions described by some known sufficient optimality conditions.

There are many papers dealing with sufficient optimality conditions for SIP and generalized SIP (see, for example [2, 14, 15, 27], etc.). In [15], and some other papers, the modified Lagrange multiplier conditions for convex problems (finite and infinite) were suggested which permit to weaken the known CQs or even to avoid them. The important property of these conditions is that they have the form of criteria and can be applied even to nonsmooth problems. Nevertheless, their practical use turns into a difficult task even for SIP problems with linear constraint functions because to check these conditions one needs to find several successions of vectors and scalars, but no constructive rules how to do it are proposed. An algorithmic implementation of the results of [15] is definitely not possible. On the contrary, in the case of convex SIP problems with linear constraints, the Implicit Optimality Criterion proved in the paper permits to formulate optimality conditions in the explicit form and to verify these conditions easily. To apply our optimality results, we need Assumption 1 to be satisfied, but as it was noticed above, this assumption is not too much restrictive.

The authors of [2] suggest the primal sufficient optimality conditions for convex SIP problems that possess a so called "uniform mean value property". To verify this property, one has to show that a certain infinite system of inequalities is inconsistent. In our paper [19], we consider convex SIP problems with one-dimensional index sets and show that even in the case when the uniform mean value property is satisfied, it is not so easy to verify the conditions from [2]. 
Evidently these conclusions are valid for the case of SIP problems with multidimensional index sets.

Now let us summarize the optimality results in the dual form suggested in [14], [27], etc., and reformulate them for the convex SIP problem in the form (10).

Suppose that $x^{0}$ is a feasible solution of problem (10). Denote

$$
K\left(x^{0}\right)=\left\{\xi \in \mathbb{R}^{n}: \xi^{T} \frac{\partial c\left(x^{0}\right)}{\partial x} \leq 0, \xi^{T} \frac{\partial f\left(x^{0}, t\right)}{\partial x} \leq 0, t \in T_{a}\left(x^{0}\right)\right\} .
$$

Consider a subset

$$
\left\{t_{j}, j=1, \ldots, p\right\} \subset T_{a}\left(x^{0}\right) .
$$

For $j=1, \ldots, p$ and $\xi \in K\left(x^{0}\right)$, denote $\Psi_{j}=\left\{\eta \in L\left(t_{j}\right): \eta^{T} \frac{\partial f\left(x^{0}, t_{j}\right)}{\partial t}=0\right\}$, and

$$
F_{j}(\xi):=\max _{\eta \in \Psi_{j}}\left(\frac{1}{2} \eta^{T} \frac{\partial^{2} f\left(x^{0}, t_{j}\right)}{\partial t^{2}} \eta+\xi^{T} \frac{\partial^{2} f\left(x^{0}, t_{j}\right)}{\partial x \partial t} \eta\right) .
$$

Theorem 5. (Theorem 5.1 in [14]) Let $x^{0}$ be a feasible solution of problem (10). Suppose that for each $\xi \in K\left(x^{0}\right) \backslash\{0\}$ there exists a finite subset (57) and vector $\lambda(\xi)=\left(\lambda_{j}(\xi), j=\right.$ $0, \ldots, p), \lambda_{j}(\xi) \geq 0, j=0, \ldots, p$, such that

(i) $\lambda(\xi) \in \Lambda:=\left\{\lambda=\left(\lambda_{j}, j=0, \ldots, p\right) \neq 0: \nabla_{x} \mathcal{L}\left(x^{0}, \lambda\right)=0\right\}$, where

$$
\mathcal{L}(x, \lambda)=\lambda_{0} c(x)+\sum_{j=1}^{p} \lambda_{j} f\left(x, t_{j}\right) ;
$$

(ii) $\xi^{T} \nabla_{x x} \mathcal{L}\left(x^{0}, \lambda(\xi)\right) \xi+2 \sum_{j=1}^{p} \lambda_{j}(\xi) F_{j}(\xi)>0$.

Then $x^{0}$ is optimal to problem (10).

One can show that if a feasible solution $x^{0} \in X$ satisfies conditions of Theorem 5 , then it satisfies conditions of Theorem 4 as well. But there exist situations when for some feasible $x^{0} \in X$ the conditions of Theorem 4 are valid, while the conditions of Theorem 5 are not.

Indeed, let us consider the example (52) suggested in the previous section. Remind that we have shown that feasible solution $x^{0}$ defined in (53) satisfies conditions of Theorem 4.

Let us verify the conditions of Theorem 5 for this $x^{0}$ in the SIP problem (52). Construct the set $\Lambda$. In our case

$$
\begin{gathered}
\Lambda=\left\{\bar{\lambda}=\left(\lambda_{0}, \lambda_{1}, \lambda_{3}, \lambda_{3}\right): \bar{\lambda} \neq 0, \bar{\lambda} \geq 0, \lambda_{0} \frac{\partial c\left(x^{0}\right)}{\partial x}+\lambda_{1} \frac{\partial f_{1}\left(x^{0}, t^{(1)}\right)}{\partial x}+\lambda_{2} \frac{\partial f_{2}\left(x^{0}, t^{(2)}\right)}{\partial x}+\right. \\
\left.\lambda_{3} \frac{\partial f_{1}\left(x^{0}, t^{(3)}\right)}{\partial x}=0\right\}=\left\{\bar{\lambda}: \bar{\lambda} \neq 0, \bar{\lambda} \geq 0, \lambda_{0}\left(\begin{array}{l}
1 \\
1 \\
0 \\
0
\end{array}\right)+\lambda_{1}\left(\begin{array}{l}
0 \\
0 \\
0 \\
0
\end{array}\right)+\lambda_{2}\left(\begin{array}{l}
0 \\
1 \\
1 \\
1
\end{array}\right)+\lambda_{3}\left(\begin{array}{c}
-4+2 x_{1}^{0} \\
2 \\
\sin 2 \\
2
\end{array}\right)=0\right\},
\end{gathered}
$$

and $\Lambda$ consists of vectors $\bar{\lambda}$ such that $\lambda_{0}=\lambda_{2}=\lambda_{3}=0, \lambda_{1}>0$.

Recall that here $T_{a}\left(x^{0}\right)=\left\{t^{(1)}, t^{(2)}, t^{(3)}\right\}=\left\{(0,0)^{T},(3,0)^{T},\left(2, x_{1}^{0}\right)^{T}\right\}$. Let us construct the set $K\left(x^{0}\right)$ by formula $(56)$ :

$$
K\left(x^{0}\right)=\left\{\xi \in \mathbb{R}^{4}: \xi^{T} \frac{\partial c\left(x^{0}\right)}{\partial x} \leq 0, \xi^{T} \frac{\partial f_{1}\left(x^{0}, t^{(1)}\right)}{\partial x} \leq 0, \xi^{T} \frac{\partial f_{1}\left(x^{0}, t^{(3)}\right)}{\partial x} \leq 0, \xi^{T} \frac{\partial f_{2}\left(x^{0}, t^{(2)}\right)}{\partial x} \leq 0\right\} .
$$


Consider vector $\bar{\xi}=(-1,0,10,-10)^{T}$. Since

$$
\bar{\xi}^{T} \frac{\partial c\left(x^{0}\right)}{\partial x}=-1<0, \bar{\xi}^{T} \frac{\partial f_{1}\left(x^{0}, t^{(1)}\right)}{\partial x}=0, \bar{\xi}^{T} \frac{\partial f_{1}\left(x^{0}, t^{(3)}\right)}{\partial x} \approx-9.816<0, \bar{\xi}^{T} \frac{\partial f_{2}\left(x^{0}, t^{(2)}\right)}{\partial x}=0
$$

then $\bar{\xi} \in K\left(x^{0}\right)$. Notice that $\bar{\xi} \neq 0$ and

$$
\bar{\xi}^{T} \frac{\partial^{2} f_{1}\left(x^{0}, t^{(1)}\right)}{\partial x \partial t}=\bar{\xi}^{T}\left(\frac{\partial^{2} f_{1}\left(x^{0}, t^{(1)}\right)}{\partial x \partial t_{1}}, \frac{\partial^{2} f_{1}\left(x^{0}, t^{(1)}\right)}{\partial x \partial t_{2}}\right)=\bar{\xi}^{T}\left(\begin{array}{ll}
0 & 0 \\
1 & 0 \\
1 & 0 \\
1 & 0
\end{array}\right)=(0,0) .
$$

Denote

$$
\begin{gathered}
\bar{F}_{i}(\xi):=\max _{\eta \in \mathbb{R}^{2}}\left(\frac{1}{2} \eta^{T} \frac{\partial^{2} f_{i}\left(x^{0}, t^{(i)}\right)}{\partial t^{2}} \eta+\xi^{T} \frac{\partial^{2} f_{i}\left(x^{0}, t^{(i)}\right)}{\partial x \partial t} \eta\right), \quad i=1,2, \\
\bar{F}_{3}(\xi):=\max _{\eta \in \mathbb{R}^{2}}\left(\frac{1}{2} \eta^{T} \frac{\partial^{2} f_{1}\left(x^{0}, t^{(3)}\right)}{\partial t^{2}} \eta+\xi^{T} \frac{\partial^{2} f_{1}\left(x^{0}, t^{(3)}\right)}{\partial x \partial t} \eta\right) .
\end{gathered}
$$

Then for problem (52), Theorem 5 can be formulated as follows:

If for all $\xi \in K\left(x^{0}\right), \xi \neq 0$, the following inequality holds:

$$
0<\max _{\bar{\lambda} \in \Lambda,\|\bar{\lambda}\|=1}\left(\lambda_{1} \bar{F}_{1}(\xi)+\lambda_{2} \bar{F}_{2}(\xi)+\lambda_{3} \bar{F}_{3}(\xi)\right)
$$

then the vector $x^{0}$ given in (53) is optimal to problem (52).

From $(58)$ and $(54)$ we get $\bar{F}_{1}(\bar{\xi})=0$. Taking into account that for the given vector $\bar{\xi}$ it holds $\bar{F}_{1}(\bar{\xi})=0$, and $\lambda_{2}=\lambda_{3}=0$, we conclude that for $\bar{\xi} \in K\left(x^{0}\right), \bar{\xi} \neq 0$ condition (59) is not satisfied. That means that the sufficient optimality conditions from Theorem 5 are not satisfied for $x^{0}$. Nevertheless $x^{0}$ is optimal to problem (52) since the sufficient optimality conditions of Theorem 4 are satisfied.

Recall here that in problem (52), both immobile indices $t^{(1)}$ and $t^{(2)}$ have the minimal immobility order equal to 1 and relations (54), (55) hold for $x^{0}$. Therefore the sufficient optimality conditions obtained in the paper describe a subset of optimal solutions that is larger (in general case) than the analogous subset determined in Theorem 5.

The results of the present section show that the optimality conditions for convex SIP based on the notion of immobile indices are either more efficient or more simple to verify than some other conditions described in literature. Therefore we have reason to believe that the study of the immobile indices and their immobility orders may allow to describe more accurately the local structure of the feasible sets of convex SIP problems both with one- and multi-dimensional index sets.

In this paper we supposed that the constraint function $f(x, t), x \in \mathbb{R}^{n}, t \in T$, is linear w.r.t. $x$. The result of the paper can be generalized to constraint functions that are faithfully convex w.r.t. $x$. For this purpose it is necessary to provide a more detailed study of the properties of the convex constraint function at the immobile indices. For one-dimensional index set $T \subset \mathbb{R}$, such study is performed in [20] (see Proposition 3.7). The generalization of the results from [20] to the case $T \subset \mathbb{R}^{s}, s>1$, is the subject of a separate paper. 


\section{Acknowledgements}

This work was partially supported by the Belarusian state program of fundamental research "Convergence 1.3.01", and by Center for Research and Development in Mathematics and Applications of Universidade de Aveiro through the Portuguese Science Foundation (FCT, Fundação para a Ciência e a Tecnologia).

The authors are grateful to two anonymous referees for their comments, corrections and constructive suggestions that have helped to prepare the final version of the paper.

\section{APPENDIX}

\section{Proof of Lemma 1.}

Let us consider the immobile index set $T^{*} \subset T$. Notice that $T$ is a compact set. Suppose that the set $T^{*}$ contains infinite number of elements. Then there exists a sequence $t^{(i)} \in T^{*}$, $i=1,2, \ldots$, such that

$$
\lim _{i \rightarrow \infty} t^{(i)}=\bar{t} \in T, \quad f\left(x, t^{(i)}\right)=0 \forall x \in X, i=1,2, \ldots
$$

Let us show that $\bar{t} \in T^{*}$. Suppose the contrary: $\bar{t} \notin T^{*}$. Then according to Definition 1 there exists $\hat{x} \in X$ such that $f(\hat{x}, \bar{t})<0$. Taking into account the continuity of function $f(\bar{x}, t), t \in T$, we conclude that there exists $\varepsilon>0$ such that $f(\hat{x}, t), t \in T,\|t-\bar{t}\| \leq \varepsilon$. But this contradicts relations (60). The contradiction proves that $\bar{t} \in T^{*}$.

Since $\bar{t} \in T^{*}$ then by Assumption 1 inequalities (11) holds true and according to Corollary 1 there exists $\bar{x} \in X$ such that relations (19) are fulfilled. The relations (19) imply the existence of $\varepsilon>0$ such that $f(\bar{x}, t)<f(\bar{x}, \bar{t})=0$ for all $t \neq \bar{t}, t \in T,\|t-\bar{t}\| \leq \varepsilon$. But this again contradicts conditions (60). Hence we have proved that the set $T^{*}$ contains a finite number of elements.

Proposition 5. Given the linear SIP problem (10) with the feasible set $X$, consider the vector function

$$
x(\lambda, \varepsilon)=(1-\lambda) y(\varepsilon)+\lambda \tilde{x}=y(\varepsilon)+\lambda(\tilde{x}-y(\varepsilon)), \lambda \in[0,1],
$$

where vector $\tilde{x} \in X$ satisfies (20)-(22), and vector $y(\varepsilon)$ is an optimal solution to problem $P(\varepsilon)$. For any sufficiently small $\varepsilon>0$ there exists $\lambda(\varepsilon) \in[0,1]$ such that

$$
x(\lambda(\varepsilon), \varepsilon) \in X \text { and } \lambda(\varepsilon) \rightarrow 0 \text { as } \varepsilon \rightarrow+0 .
$$

Proof. Given a sufficiently small $\varepsilon>0$, denote $T^{+}(\varepsilon)=\{t \in T: f(y(\varepsilon), t)>0\}$. It is evident that $T^{+}(\varepsilon) \subset \bigcup_{j \in I^{*}} T_{\varepsilon}\left(t_{j}^{0}\right)$ where $\left\{t_{j}^{0}, j \in I^{*}\right\}$ is the set of immobile indices in problem (10), $T_{\varepsilon}(t)=T \cap\left\{\tau \in \mathbb{R}^{s}:\|t-\tau\| \leq \varepsilon\right\}$. Taking into account the convexity of $f(x, t)$ w.r.t. $x$ we conclude that

$$
f(x(\lambda, \varepsilon), t) \leq 0, t \in T \backslash T^{+}(\varepsilon), \text { for all } \lambda \in[0,1] .
$$

Denote

$$
\lambda_{j}(\varepsilon, t):=\frac{f(y(\varepsilon), t)}{f(y(\varepsilon), t)-f(\tilde{x}, t)}, t \in T_{\varepsilon}\left(t_{j}^{0}\right) \cap T^{+}(\varepsilon), j \in I^{*} .
$$


It is evident that

$$
f(x(\lambda, \varepsilon), t) \leq 0 \text { if } \lambda \geq \lambda_{j}(\varepsilon, t), t \in T_{\varepsilon}\left(t_{j}^{0}\right) \cap T^{+}(\varepsilon), j \in I^{*} .
$$

Let us fix (arbitrary) $j \in I^{*}$, and estimate the corresponding values of $\lambda_{j}(\varepsilon, t), t \in T_{\varepsilon}\left(t_{j}^{0}\right) \cap T^{+}(\varepsilon)$. By construction $f(y(\varepsilon), t)>0, t \in T_{\varepsilon}\left(t_{j}^{0}\right) \cap T^{+}(\varepsilon)$, hence we can conclude that

$$
\lambda_{j}(\varepsilon, t) \leq \frac{f(y(\varepsilon), t)}{-f(\tilde{x}, t)}, t \in T_{\varepsilon}\left(t_{j}^{0}\right) \cap T^{+}(\varepsilon)
$$

Set

$$
\begin{gathered}
c_{j}(\varepsilon):=\frac{\partial f\left(y(\varepsilon), t_{j}^{0}\right)}{\partial t}, \tilde{c}_{j}:=\frac{\partial f\left(\tilde{x}, t_{j}^{0}\right)}{\partial t}, \quad S_{j}(\varepsilon):=\frac{\partial^{2} f\left(y(\varepsilon), t_{j}^{0}\right)}{\partial t^{2}}, \tilde{S}_{j}:=\frac{\partial^{2} f\left(\tilde{x}, t_{j}^{0}\right)}{\partial t^{2}} \\
D_{j}(\varepsilon, l):=\left.\frac{\partial^{3} f\left(y(\varepsilon), t_{j}^{0}+\alpha l\right)}{\partial \alpha^{3}}\right|_{\alpha=+0} .
\end{gathered}
$$

The index $t \in T_{\varepsilon}\left(t_{j}^{0}\right) \cap T^{+}(\varepsilon)$ can be presented in the form $t=t_{j}^{0}+\Delta t_{j} l$ where $l \in L\left(t_{j}^{0}\right)$ is a feasible direction in $t_{j}^{0}$, and $0 \leq \Delta t_{j} \leq \varepsilon$. Notice that every $l \in L\left(t_{j}^{0}\right)$ can be written in the form $l=\gamma l_{1}+\omega l_{2}$ with

$$
\gamma^{2}+\omega^{2}=1, \gamma \geq 0, \omega \geq 0, l_{1}=N_{j} \alpha, l_{2}=M_{j} \beta, \alpha \geq 0,\|\alpha\|=1,\|\beta\|=1,
$$

the matrices $M_{j}$ and $N_{j}$ being defined in (29).

Then taking into account that by construction (see (20)-(22), and (32)) it holds

$$
f\left(y(\varepsilon), t_{j}^{0}\right)=0, c_{j}^{T}(\varepsilon) l_{2}=0, f\left(\tilde{x}, t_{j}^{0}\right)=0, \tilde{c}_{j}^{T} l_{2}=0,
$$

we can write for $t \in T_{\varepsilon}\left(t_{j}^{0}\right) \cap T^{+}(\varepsilon)$ the following expansions:

$$
\begin{gathered}
f(y(\varepsilon), t)=f\left(y(\varepsilon), t_{j}^{0}+\Delta t_{j} l\right)=\Delta t_{j} c_{j}^{T}(\varepsilon) l+\frac{\Delta t_{j}^{2}}{2} l^{T} S_{j}(\varepsilon) l+\frac{\Delta t_{j}^{3}}{3 !} D_{j}(\varepsilon, l)+o\left(\Delta t_{j}^{3}\right)= \\
\Delta t_{j} \gamma c_{j}^{T}(\varepsilon) l_{1}+\frac{\Delta t_{j}^{2}}{2}\left(\gamma^{2} l_{1}^{T} S_{j}(\varepsilon) l_{1}+2 \gamma \omega l_{1}^{T} S_{j}(\varepsilon) l_{2}+\omega^{2} l_{2}^{T} S_{j}(\varepsilon) l_{2}\right)+\frac{\Delta t_{j}^{3}}{3 !} D_{j}(\varepsilon, l)+o\left(\Delta t_{j}^{3}\right), \\
f(\tilde{x}, t)=f\left(\tilde{x}, t_{j}^{0}+\Delta t_{j} l\right)=\Delta t_{j} \tilde{c}_{j}^{T} l+\frac{\Delta t_{j}^{2}}{2} l^{T} \tilde{S}_{j} l+o\left(\Delta t_{j}^{2}\right)= \\
\Delta t_{j} \gamma \tilde{c}_{j}^{T} l_{1}+\frac{\Delta t_{j}^{2}}{2}\left(\gamma^{2} l_{1}^{T} \tilde{S}_{j} l_{1}+2 \gamma \omega l_{1}^{T} \tilde{S}_{j} l_{2}+\omega^{2} l_{2}^{T} \tilde{S}_{j} l_{2}\right)+o\left(\Delta t_{j}^{2}\right)= \\
\Delta t_{j} \gamma \tilde{c}_{j}^{T} l_{1}+\frac{\Delta t_{j}^{2}}{2} l_{2}^{T} \tilde{S}_{j} l_{2}+\frac{\Delta t_{j}^{2}}{2} \gamma\left(\gamma l_{1}^{T} \tilde{S}_{j} l_{1}+2 \omega l_{1}^{T} \tilde{S}_{j} l_{2}-\gamma l_{2}^{T} \tilde{S}_{j} l_{2}\right)+o\left(\Delta t_{j}^{2}\right) .
\end{gathered}
$$

Now recall that by construction (see again (32)) the following inequalities take place:

$$
c_{j}^{T}(\varepsilon) l_{1} \leq 0, l_{2}^{T} S_{j}(\varepsilon) l_{2} \leq 0 .
$$

Then in (65) we get

$$
\begin{aligned}
& f(y(\varepsilon), t) \leq \frac{\Delta t_{j}^{2}}{2}\left(\gamma^{2} l_{1}^{T} S_{j}(\varepsilon) l_{1}+2 \gamma \omega l_{1}^{T} S_{j}(\varepsilon) l_{2}\right)+\frac{\Delta t_{j}^{3}}{3 !} D_{j}(\varepsilon, l)+o\left(\Delta t_{j}^{3}\right) \\
& \leq \Delta t_{j}^{2}\left[\frac{1}{2}\left(\gamma^{2}\left|l_{1}^{T} S_{j}(\varepsilon) l_{1}\right|+2 \gamma \omega\left|l_{1}^{T} S_{j}(\varepsilon) l_{2}\right|\right)+\frac{\Delta t_{j}}{3 !}\left|D_{j}(\varepsilon, l)\right|+o\left(\Delta t_{j}\right)\right] .
\end{aligned}
$$

Denote $\mu=\max \left\{\Delta t_{j}, \gamma\right\}$ and notice that

$$
0 \leq \frac{\gamma}{\mu} \leq 1, \quad 0 \leq \frac{\Delta t_{j}}{\mu} \leq 1, \quad 0 \leq \gamma \leq 1, \quad 0 \leq \omega \leq 1
$$


With respect to the latest inequalities, we get from (67) and (66) that for $t \in T_{\varepsilon}\left(t_{j}^{0}\right) \cap T^{+}(\varepsilon)$ it holds

$$
\begin{gathered}
\frac{1}{\mu \Delta t_{j}^{2}} f(y(\varepsilon), t) \leq \frac{1}{2}\left(\gamma \frac{\gamma}{\mu}\left|l_{1}^{T} S_{j}(\varepsilon) l_{1}\right|+2 \frac{\gamma}{\mu} \omega\left|l_{1}^{T} S_{j}(\varepsilon) l_{2}\right|\right)+\frac{\Delta t_{j}}{3 ! \mu}\left|D_{j}(\varepsilon, l)\right|+O\left(\Delta t_{j}\right) \\
\leq\left|l_{1}^{T} S_{j}(\varepsilon) l_{1}\right| / 2+\left|l_{1}^{T} S_{j}(\varepsilon) l_{2}\right|+\left|D_{j}(\varepsilon, l)\right| / 3 !+O\left(\Delta t_{j}\right), \\
\frac{-1}{\mu \Delta t_{j}} f(\tilde{x}, t)=-\left[\frac{\gamma}{\mu} \tilde{c}_{j}^{T} l_{1}+\frac{\Delta t_{j}}{2 \mu} l_{2}^{T} \tilde{S}_{j} l_{2}+\Delta t_{j} \frac{\gamma}{2 \mu}\left(\gamma l_{1}^{T} \tilde{S}_{j} l_{1}+2 \omega l_{1}^{T} \tilde{S}_{j} l_{2}-\gamma l_{2}^{T} \tilde{S}_{j} l_{2}\right)+\frac{o\left(\Delta t_{j}\right)}{\mu}\right] \\
=-\left[\frac{\gamma}{\mu} \tilde{c}_{j}^{T} l_{1}+\frac{1}{2} \frac{\Delta t_{j}}{\mu} l_{2}^{T} \tilde{S}_{j} l_{2}+O\left(\Delta t_{j}\right)\right] \geq \min \left\{-\tilde{c}_{j}^{T} l_{1},-\frac{1}{2} l_{2}^{T} \tilde{S}_{j} l_{2}\right\}+O\left(\Delta t_{j}\right) .
\end{gathered}
$$

Evidently (64), (68), (69) imply the inequalities

$$
\lambda_{j}(\varepsilon, t) \leq \Delta t_{j} \frac{\left|l_{1}^{T} S_{j}(\varepsilon) l_{1}\right| / 2+\left|l_{1}^{T} S_{j}(\varepsilon) l_{2}\right|+\left|D_{j}(\varepsilon, l)\right| / 3 !+O\left(\Delta t_{j}\right)}{\min \left\{-\tilde{c}_{j}^{T} l_{1},-\frac{1}{2} l_{2}^{T} \tilde{S}_{j} l_{2}\right\}+O\left(\Delta t_{j}\right)}, t \in T_{\varepsilon}\left(t_{j}^{0}\right) \cap T^{+}(\varepsilon) .
$$

Since by construction

$$
\begin{gathered}
\tilde{c}_{j}^{T} l_{1}<0 \text { for all } l_{1}=N_{j} \alpha, \alpha \geq 0,\|\alpha\|=1, \alpha \in \mathbb{R}^{r_{j}}, \\
l_{2}^{T} \tilde{S}_{j} l_{2}<0 \text { for all } l_{2}=M_{j} \beta, \beta \in \mathbb{R}^{s_{j}},\|\beta\|=1,
\end{gathered}
$$

then

$$
\tilde{a}_{j}:=\min \left\{a_{j}, b_{j} / 2\right\}>0
$$

where

$$
\begin{gathered}
a_{j}=\min _{l_{1}, \alpha}-\tilde{c}_{j}^{T} l_{1}, \quad \text { s.t. } l_{1}=N_{j} \alpha, \alpha \geq 0,\|\alpha\|=1, \alpha \in \mathbb{R}^{r_{j}}, \\
b_{j}:=\min _{l_{2}, \beta}-l_{2}^{T} \tilde{S}_{j} l_{2}, \quad \text { s.t. } l_{2}=M_{j} \beta, \beta \in \mathbb{R}^{s_{j}},\|\beta\|=1 .
\end{gathered}
$$

Moreover we have $0 \leq \tilde{b}_{j}<\infty$, where

$$
\begin{gathered}
\tilde{b}_{j}:=\max _{l_{1}, l_{2}, y}\left(\left|l_{1}^{T} S_{j}(y) l_{1}\right| / 2+\left|l_{1}^{T} S_{j}(y) l_{2}\right|+\left|D_{j}(y, l)\right| / 3 !\right), \\
\text { s.t. }\left\|l_{1}\right\|=1,\left\|l_{2}\right\|=1,\|l\|=1,\left\|y-x^{0}\right\| \leq \varepsilon_{0} .
\end{gathered}
$$

Here $S_{j}(y):=\frac{\partial^{2} f\left(y, t_{j}^{0}\right)}{\partial t^{2}}, D_{j}(y, l):=\left.\frac{\partial^{3} f\left(y, t_{j}^{0}+\alpha l\right)}{\partial \alpha^{3}}\right|_{\alpha=+0}, j \in I^{*}$.

It follows from (70) and the last relations that for sufficiently small $\varepsilon>0$ the following inequalities are satisfied:

$$
\lambda_{j}(\varepsilon, t) \leq \varepsilon\left(\lambda_{j}+1\right), \quad t \in T_{\varepsilon}\left(t_{j}^{0}\right) \cap T^{+}(\varepsilon) \text { with } \lambda_{j}=\frac{\tilde{b}_{j}}{\tilde{a}_{j}}, 0 \leq \lambda_{j}<\infty .
$$

Notice that estimations (71) can be proved for all $j \in I^{*}$. Hence these estimations and relations (63) imply the inequalities

$$
\begin{gathered}
f(x(\lambda(\varepsilon), \varepsilon), t) \leq 0, t \in T_{\varepsilon}\left(t_{j}^{0}\right) \cap T^{+}(\varepsilon), j \in I^{*}, \\
\text { with } \lambda(\varepsilon):=\varepsilon\left(\lambda_{*}+1\right), \lambda_{*}=\max _{j \in I^{*}} \lambda_{j} .
\end{gathered}
$$

Finally, relations (61) follow from (62) and (72), and the proposition is proved. 
Corollary 3. The following equality takes place

$$
\lim _{\varepsilon \rightarrow+0} c(y(\varepsilon))=c\left(x^{0}\right)
$$

where $y(\varepsilon)$ is an optimal solution to problem $(P(\varepsilon))$.

Proof. Taking into account (33), the inclusion $x(\lambda(\varepsilon), \varepsilon) \in X$ proved in Proposition 5, and the convexity of $c(x)$,we get

$$
c(y(\varepsilon)) \leq c\left(x^{0}\right) \leq c(x(\lambda(\varepsilon), \varepsilon)) \leq c(y(\varepsilon))+\lambda(\varepsilon)(c(\tilde{x})-c(y(\varepsilon))) .
$$

Let us pass on to the limit in the latest relations. As a result we have $\lim _{\varepsilon \rightarrow+0} c(y(\varepsilon)) \leq c\left(x^{0}\right) \leq$ $\lim _{\varepsilon \rightarrow+0} c(y(\varepsilon))$. Consequently, equality (73) takes place. The corollary is proved.

Proposition 6. Given the linear SIP problem (10), consider the vector-function $x(\lambda)=(1-$ $\lambda) y+\lambda \tilde{x}, \lambda \in[0,1]$, where vector $\tilde{x}$ satisfies (20)-(22) and vector $y$ satisfies (28) for some $j \in I^{*}$. Then for $\forall \lambda \in(0,1]$ there exists a number $\Delta=\Delta(\lambda)>0$ such that

$$
f(x(\lambda), t) \leq 0, t \in T_{\Delta}\left(t_{j}^{0}\right) .
$$

Proof. Let $\bar{\Delta}(\lambda) \geq 0$ be the maximal number $\Delta$ such that conditions (74) hold. It is evident that $\bar{\Delta}(\bar{\lambda}) \geq \bar{\Delta}(\lambda)$ if $\bar{\lambda}>\lambda$. Consequently, to prove the theorem, it is enough to show that relations (74) take place for sufficiently small $\lambda>0$.

Repeating the reasonings used in the proof of Proposition 5, we conclude that the following relations hold:

$$
f(x(\lambda), t)=f\left(x(\lambda), t_{j}^{0}+\Delta t_{j} l\right) \leq 0 \quad \forall \Delta t_{j} \in[0, \varepsilon], \forall l \in L\left(t_{j}^{0}\right),
$$

if the numbers $\lambda>0$ and $\varepsilon>0$ satisfy the equality (see (72)) $\lambda=\varepsilon\left(\lambda_{*}+1\right)$ where $0 \leq \lambda_{*}<\infty$, $\varepsilon$ is sufficiently small. Consequently, for sufficiently small $\lambda>0$ and $\varepsilon=\varepsilon(\lambda):=\frac{\lambda}{\lambda_{*}+1}>0$, relations (75) take place. These relations will result in relations (74) if we put $\Delta=\Delta(\lambda)=\varepsilon(\lambda)>0$. The proposition is proved.

\section{References}

[1] Abrams R. and Kerzner L. A simplified test for optimality. JOTA, vol.25, N1 (1978), pp.161-170.

[2] Ben Tal A., Kerzner L., and Zlobec S. Optimality conditions for convex semi-infinite programming problems. Naval Res. Logist. 27, N.3 (1980), pp. 413-435.

[3] Bonnans J.F., Shapiro A. Perturbation analysis of optimization problems. Springer-Verlag, New-York, 2000.

[4] Borwein J. M. and Wolkowicz H. Characterizations of optimality without constraint qualification for the abstract convex program. Mathematical Programming study, 19 (1982), pp. 77-100. North-Holland Publishing Company. 
[5] Borwein J. M. and Wolkowicz H. Regularizing the abstract convex program. J. Math. Anal. Appl., 83 (1981), pp. 495-530.

[6] Charnes A., Cooper W.W., and Kortanek K.O. On the theory of semi-infinite programming and some generalizations of Kuhn-Tucker saddle point theorems for arbitrary convex functions. Naval Res. Logist., 16 (1969), pp.41-51.

[7] Fajardo M.D. and López M.A. Some results about the facial geometry of convex semiinfinite systems. Optimization V.55, N.5-6 (2006), pp.661-684.

[8] Goberna M.A., Jeyakumar V., and López M.A. Necessary and sufficient constraint qualifications for systems of infinite convex inequalities. J. Nonlinear Anal., 68 (2008), pp.11841194.

[9] Goberna M.A., López M.A. (eds.) Semi-infinite programming: recent advances. Kluwer, Dordrecht, 2001.

[10] Gruber G., Kruk S., Rendl F., and Wolkowicz H. Presolving for semidefinite program without constraint qualifications. In G. Gruber et al. editor, Proceedings of HPOP97, Second Workshop on High Perfomance Optimization Techniques, Rotterdam Netherlands, (1997).

[11] Gustafson S.-A. On semi-infinite programming in numerical analysis. In Semi-Infinite Programming. Lecture Notes in Control and Inf. Sci., 15, R.Hettich ed., Springer-Verlag, Berlin, Heidelberg, New York (1979) pp. 137-153.

[12] Hettich R., Kortanek K.O. Semi-infinite programming: theory, methods and applications. SIAM Rev., 35 (1993) pp. 380-429.

[13] Hettich R., Jongen H. Th. Semi-infinite programming: conditions of optimality and applications. In J. Stoer, ed., Optimization Techniques, Part 2, Lecture Notes in Control and Information Sciences N.7 (1978) pp. 1-11.

[14] Hettich R., Still G. Second order optimality conditions for generalized semi-infinite programming problems. Optimization, 34 (1995), pp. 195-211.

[15] Jeyakumar V., Lee G.M., and Dihn N. New sequential Lagrange multiplier conditions characterizing optimality without constraint qualification for convex programs. SIAM J. Optim., V.14, N. 2 (2003) pp.534-547.

[16] Jongen H. Th., Wetterling W., and Zwier G. On sufficient conditions for local optimality in semi-infinite programming. Optimization, 18 (1987) pp. 165-178.

[17] Klatte D. Stable local minimizers in semi-infinite optimization: regularity and second-order conditions. J. Comput. Appl. Math 56, Issues 1-2 (1994) pp. 137-157.

[18] Kortanek K.O. and Medvedev V.G. Semi-infinite programming and new applications in finance. In: C.Floudas and Panos Pardalos, Editors, Encyclopedia of Optimization. Kluwer Academic, 2005.

[19] Kostyukova O.I. and Tchemisova T.V. Sufficient optimality conditions for convex semiinfinite programming. Optimization Methods and Software, V. 25, Issue 2 (2010) pp. 279297. 
[20] Kostyukova O.I., Tchemisova T.V., and Yermalinskaya S.A. On the algorithm of determination of immobile indices for convex SIP problems. IJAMAS-International Journal on Mathematics and Statistics, 13, N. J08 (2008) p.13-33.

[21] Kostyukova O.I., and Tchemisova T.V. Optimality criteria without constraint qualification for linear semidefinite problems, accepted to publication in the special issue of JMS "Algebraic Techniques in Graph Theory and Optimization", 2010.

[22] Li. W., Nahak Ch., and Singer I. Constraint qualifications for semi-infinite systems of convex inequalities. SIAM J. Optim., V. 11, N 1 (2000) pp. 31-52.

[23] Polak E. Semi-infinite optimization in engineering design. In A.V. Fiacco and K.O. Kortanek, Editors, Semi-Infinite Programming and Applications, Lecture Notes in Econom. and Math. Systems. Springer-Verlad (1983) pp.236-248.

[24] Ramana M. V. An exact duality theory for semidefinite programming and its complexity implications. DIMACS Technical report 95-02R, RUTCOR, Rutgers University, New Brunswick, NJ, 1995.

[25] Rückmann J. J. and Shapiro A. First-order optimality conditions in generalized semiinfinite programming. J. of Optimization Theory and Appl., V. 101, No.3 (1999) pp.677691.

[26] Rückmann J.J. and Shapiro A. Second-order optimality conditions in generalized semiinfinite programming. Set-Valued Anal., 9, Numbers 1-2 (2001) pp. 169-186.

[27] Stein O. and Still G. On optimality conditions for generalized semi-infinite programming problems. J. Optim. Theory Appl., 104, N.2 (2000) pp. 443-458.

[28] Still G. Generalized semi-infinite programming: theory and methods. EJOR, Vol.119 (1999) pp.301-313

[29] Tijs S.H. Semi-infinite linear programs and semi-infinite matrix games. Nieuw Arch. Wisk. (1979) 27.

[30] Wolkowicz H. Method of reduction in convex programming. JOTA, V. 40, N.3 (1983) pp.349378.

[31] Wolkowicz H., Saigal R., and Vandenberghe L.(editors) Handbook of semidefinite programming: theory, algorithms, and applications, edited by Kluwer Academic Publishers, 2000. 